\title{
APLICATION OF A NEW OBSERVATIONAL STRATEGY TO THE STUDY OF GRAVITATIONAL SOLAR MODES.
}

\author{
I. MARTIN MATEOS (IMM@IAC.ES) AND P.L. PALLÉ \\ Instituto de Astrofísica de Canarias
}

\begin{abstract}
.
The aim of the present work is the detection of solar g-modes, making use of their spatial and temporal properties, by means of a new observational strategy. The basic data, gathered at the Observatorio del Teide in 1993, consists on daily solar velocity measurements taken continuous and sequentially at six different and symmetric positions on the solar disk. By correlating the time series obtained from different positions, and considering the geometrical properties of different modes $(l, m)$ on the Sun's surface, some of them can selectively be eliminated or enhanced. In particular, the main spectral features present in the resulting power spectra must have precise phase relations if they correspond to global solar g-modes.
\end{abstract}

\section{Observations and data.}

The data available for the analysis were obtained using a resonant spectrophotometer (long stability). This instrument measures the line of sight velocity in the potassium 7699 A line.

The data are obtained with a new strategy using alternative and continuous measurements at 6 different positions, uniformly distributed on the solar disk. The advantage of observing at the solar periphery is to increase the horizontal velocity signal, which theoretical predictions suppose is the highest for g-modes. Since the global modes have different temporal phase at different apertures, we used this property to identify and classify modes, combining measurements from different positions. The combinations let us filter out the signal from some particular modes and to enhance others. The 6 apertures are separated by 60 degrees with the centers at $0.55 R_{\odot}$. The measurements are taken alternatively each 20 seconds, with a cycle continued during all the daily observations. Many spectra have been obtained from these series of data, from individual apertures and from the combination of them, by FFT methods.

\section{Peak selection.}

The work has been performed on the interval $50-250 \mu \mathrm{Hz}$. The peak selection process seeks for common peaks in independent spectra combinations. First, the method looks 
for peaks whose amplitude is more than a threshold $(2 \sigma, 2.5 \sigma$ and $3 \sigma$; being $\sigma$ the standard deviation). At this time only peaks which have passed the threshold limit are considered. For the selection of the standard deviation the frequency interval was divided into ten small intervals, with $20 \mu \mathrm{Hz}$ each. Different standard deviations were considered because the noise level is strongly variable in the whole interval. The next step was to study the phase, and several peaks were selected, those which presented a high amplitude and the same phase (within limits of either 10 or 20 degrees) in various combinations of the series.

Finally we checked the time evolution of the selected peaks, and different tests were applied for studying their properties. The following table shows the final results, with the basic properties of the selected peaks. These peaks are the candidates to $\mathrm{g}$-mode frequency peaks.

TABLE 1. Selected peaks. Resolution $0.01 \mu \mathrm{Hz}$.

\begin{tabular}{|c|c|c|c|}
\hline Frequency $(\mu H z)$ & Period (min.) & Amplitude $(\mathrm{cm} / \mathrm{s})$ & noise $(\mathrm{cm} / \mathrm{s})$ \\
\hline 53.35 & 312.4 & 107.69 & 7.5 \\
\hline 58.39 & 285.43 & 60.01 & 4.52 \\
\hline 59.28 & 281.4 & 67.65 & 4.52 \\
\hline 70.35 & 236.9 & 103.94 & 6.16 \\
\hline 70.79 & 235.43 & 75.94 & 4.54 \\
\hline 71.08 & 234.47 & 96.08 & 6.16 \\
\hline 79.79 & 208.88 & 121.76 & 6.16 \\
\hline 105.13 & 158.53 & 67.94 & 5.87 \\
\hline 114.03 & 146.16 & 62.18 & 4.77 \\
\hline 116.75 & 142.75 & 45.89 & 4.77 \\
\hline 129.41 & 128.78 & 45.68 & 4.77 \\
\hline 130.54 & 125.58 & 28.67 & 3.57 \\
\hline 135.91 & 122.60 & 26.65 & 3.57 \\
\hline 164.14 & 101.53 & 38.57 & 2.98 \\
\hline 170.65 & 97.66 & 31.42 & 2.96 \\
\hline 182.57 & 91.28 & 24.81 & 2.96 \\
\hline 194.16 & 85.83 & 35.17 & 2.91 \\
\hline 232.41 & 70.53 & 18.35 & 2.46 \\
\hline
\end{tabular}

